\title{
Christina Victor, Sasha Scambler and John Bond (2009). The Social World of Older People. Understanding Loneliness and Social Isolation in Later Life. Maidenhead: Open University Press, 262 pp. ISBN 9780335215218 (paperback)
}

\author{
REVIEWED BY LARS ANDERSSON*
}

Every once in a while it is useful to have updates of central research areas. Hence, the book by Victor, Scambler and Bond is a timely and welcome contribution to the research on loneliness and kindred phenomena.

Unfortunately, the book also functions as a summary report of one of the projects in the Growing Older Program funded by the Economic and Social Research Council, which is of less interest to the general reader. The book offers a detailed account of loneliness and social isolation among older people, particularly in Britain. The latter specification, of course, is a limitation for an international audience; however, having said that, the volume nevertheless is very useful for those interested in these issues.

As outlined in the Introduction, the specific objectives have been to describe the prevalence of social isolation and loneliness and to contribute (1) to our understanding of secular trends in the experience of quality of life (2), to knowledge and understanding of later life by investigating the relationship between loneliness and social isolation and living alone, and (3) to contribute to policy and practice by identifying the factors, resources and coping mechanisms that protect older people from experiencing

* Lars Andersson, NISAL, Linköping University, Sweden 
International Journal of Ageing and Later Life

loneliness and isolation. Having the seminal work of Townsend, in particular, as exemplar, one stated ambition has been to argue for the revival of the explicitly sociological contribution to the analysis of older people's social worlds.

Following the Introduction, Chapter 2 elucidates theoretical and methodological issues with regard to loneliness and social isolation. It includes an exposition of various measures of loneliness that have been developed over the years, which is instructive given that the reason for the choice of measure is seldom qualified in articles. In the same chapter, the reader is also introduced to the authors' own study, which is comprised of a quantitative survey of 999 individuals aged 65 and above and a qualitative study with 45 participants. The presentation of the studies exposes some problems and lack of clarity. One such problem is the low response rate in the quantitative study (63\%). It surely has some implication for the results. Another problem, or lack of clarity, is that the authors claim to challenge the objective nature of loneliness and social isolation (p. 38), while in Chapter 3, essentially objective measures are presented from the quantitative study (cf. Tables 3.1 through 3.4). As the questionnaire is not included, it is not possible to get a complete picture of the measures. In Chapter 4, the main focus is on loneliness, and can be seen as a continuation of Chapter 2. Here, comparisons are made with several both British and international studies. To get some indication of whether people "tell the truth" about loneliness, a comparison was made between the answers to the single item question in the quantitative study and the way the (same) participants talked about loneliness in the qualitative interview. The agreement turned out to be quite good.

In Chapter 5, the focus is shifted to social exclusion and inclusion and their relationships to social networks. Based on the results from the qualitative study, the authors note that isolation is conceptualised by older people as having a strong spatial or neighbourhood component. In order to facilitate historical comparison a social contact score was generated. The results of the comparisons have to be interpreted carefully. In regard to comparative studies, the authors earlier (p. 74) noted the difficulty of comparing older populations over time as the composition, for example the proportions of marital status and of people living alone, differ 
substantially. The phenomenon of, for example, living alone may also have shifted meaning over the years.

In spite of all the data, the book does not add many new thoughts on the issue. The outcome merely confirms what we already know. Maybe the ambition to partly make a "follow up" of the classic studies by Townsend and others has restricted the design of the studies. It is concluded in the final Chapter (6) that the levels of severe isolation and loneliness have remained remarkably constant over time, and that the authors do not see vast increase in levels of loneliness and isolation in future decades; that the lonely can be divided into a variety of differing subgroups; that the telephone and the mobile phone enable families and friends to stay in touch; that a variety of different pathways lead to loneliness and isolation, and finally that loneliness and isolation will remain an experience that is confined to a minority of older people. Hence, most disappointing with the book is that the authors did not take the opportunity to take the area of loneliness and social isolation further by way of their own studies.

I acclaim the authors' sociological approach. However, it seems to have a restricting effect on their analysis in that their starting point is that isolation and loneliness can only be understood by locating them within the broader context of relationships (pp. 4-5). My standpoint is quite the opposite. I have become critical of the obsession with social contacts, social isolation or social networks in connection with loneliness. 